\title{
A NOTE ON BILINEAR WAVE-SCHRÖDINGER INTERACTIONS
}

\author{
TIMOTHY CANDY
}

\begin{abstract}
We consider bilinear restriction estimates for wave-Schrödinger interactions and provided a sharp condition to ensure that the product belongs to $L_{t}^{q} L_{x}^{r}$ in the full bilinear range $\frac{2}{q}+\frac{d+1}{r}<d+1,1 \leqslant q, r \leqslant 2$. Moreover, we give a counter-example which shows that the bilinear restriction estimate can fail, even in the transverse setting. This failure is closely related to the lack of curvature of the cone. Finally we mention extensions of these estimates to adapted function spaces. In particular we give a general transference type principle for $U^{2}$ type spaces that roughly implies that if an estimate holds for homogeneous solutions, then it also holds in $U^{2}$. This transference argument can be used to obtain bilinear and multilinear estimates in $U^{2}$ from the corresponding bounds for homogeneous solutions.
\end{abstract}

Let $u=e^{i t|\nabla|} f$ be a free wave, and let $v=e^{i t \Delta} g$ be a homogeneous solution to the Schrödinger equation. Our goal is to understand for which $1 \leqslant q, r \leqslant \infty$ we have the bilinear estimate

$$
\|u v\|_{L_{t}^{q} L_{x}^{r}\left(\mathbb{R}^{1+d}\right)} \lesssim\|f\|_{L^{2}\left(\mathbb{R}^{d}\right)}\|g\|_{L^{2}\left(\mathbb{R}^{d}\right)} .
$$

As a first step in this direction, assuming for instance that we have the support condition supp $\widehat{f}$, supp $\widehat{g} \subset$ $\{|\xi| \approx 1\}$, then for any $\frac{2}{q_{1}}+\frac{d-1}{r_{1}} \leqslant \frac{d-1}{2}$ with $\left(q_{1}, r_{1}, d\right) \neq(2, \infty, 3)$, and any $\frac{2}{q_{2}}+\frac{d}{r_{2}} \leqslant \frac{d}{2}$ with $\left(q_{2}, r_{2}, d\right) \neq$ $(2, \infty, 2)$ we have the linear Strichartz estimates

$$
\|u\|_{L_{t}^{q_{1}} L_{x}^{r_{1}\left(\mathbb{R}^{1+d}\right)}} \lesssim\|f\|_{L^{2}\left(\mathbb{R}^{d}\right)}, \quad\|v\|_{L_{t}^{q_{2}} L_{x}^{r_{2}\left(\mathbb{R}^{1+d}\right)}} \lesssim\|g\|_{L^{2}\left(\mathbb{R}^{d}\right)}
$$

Consequently an application of Hölder's inequality and a short computation shows that the bilinear estimate (1) holds provided that

$$
\frac{2}{q}+\frac{d}{r} \leqslant d, \quad \frac{2}{q}+\frac{d-1}{r} \leqslant d-1+\frac{1}{d}, \quad \text { and } \quad(q, d) \neq\left(\frac{4}{3}, 2\right),(1,3) .
$$

The first condition in (2) is stronger in the region $q \geqslant 2$ and follows by simply placing $u \in L_{t}^{\infty} L_{x}^{2}$ and using the Strichartz estimate for $v$. Note that this explains the Schrödinger scaling of the first condition in (2). The second condition in (2) dominates in the region $1 \leqslant q \leqslant 2$, where we are forced to use the Strichartz estimates on both $u$ and $v$.

A natural question now arises, is it possible to improve on the conditions (2)? This question is particularly relevant in applications to nonlinear PDE, where bilinear estimates such as (2) with $q, r$ as small as possible, are extremely useful in controlling nonlinear interactions. Note that the wave-Schrödinger interactions occur naturally in important models, see for instance the Zakharov system [17. In the case of wave-wave interactions, it is possible to improve significantly on the range given by simply applying Hölder's inequality and the Strichartz estimate for the wave equation provided an additional transversality assumption is made.

Theorem 1 (Bilinear restriction for wave [16, 14]). Let $d \geqslant 2$ and $1 \leqslant q, r \leqslant 2$ with $\frac{2}{q}+\frac{d+1}{r}<d+1$. If $f, g \in L^{2}\left(\mathbb{R}^{d}\right)$ and $\omega, \omega^{\prime} \in \mathbb{S}^{d-1}$ with $\prod^{1} \measuredangle\left(\omega, \omega^{\prime}\right) \approx 1$ and

$$
\operatorname{supp} \widehat{f} \subset\left\{\xi \in \mathbb{R}^{d}|| \xi \mid \approx 1, \measuredangle(\xi, \omega) \ll 1\right\}, \quad \operatorname{supp} \widehat{g} \subset\left\{\xi \in \mathbb{R}^{d}|| \xi \mid \approx 1, \measuredangle\left(\xi, \omega^{\prime}\right) \ll 1\right\}
$$

then

$$
\left\|e^{i t|\nabla|} f e^{i t|\nabla|} g\right\|_{L_{t}^{q} L_{x}^{r}\left(\mathbb{R}^{1+d}\right)} \lesssim\|f\|_{L^{2}\left(\mathbb{R}^{d}\right)}\|g\|_{L^{2}\left(\mathbb{R}^{d}\right)}
$$

Financial support by the Marsden Fund Council grant 19-UOO-142, and the German Research Foundation (DFG) through the CRC 1283 "Taming uncertainty and profiting from randomness and low regularity in analysis, stochastics and their applications" is acknowledged.

${ }^{1}$ Here $\measuredangle(x, y)=\left(1-\frac{x \cdot y}{|x||y|}\right)^{\frac{1}{2}}$ is the angle between $x, y \in \mathbb{R}^{d} \backslash\{0\}$. 
The first result beyond the linear Strichartz theory was obtained in [5]. The endpoint and extension to more general frequency interactions is also known [13, 14, 15]. The range for $(q, r)$ is sharp, and was originally conjectured by Klainerman-Machedon. Theorem 1 is closely related to the restriction conjecture for the cone, as the free wave $e^{i t|\nabla|} f$ is essentially the extension operator for the cone. In particular, bilinear estimates of the form (1) were originally used to obtain restriction estimates for the cone, see for instance [12.

Theorem 1 is truly a bilinear estimate as it relies crucially on the support assumption (3). This assumption implies that the two subsets of the cone, $\operatorname{supp} \mathcal{F}\left[e^{i t|\nabla|} f\right] \subset \mathbb{R}^{1+d}$ and supp $\mathcal{F}\left[e^{i t|\nabla|} g\right] \subset \mathbb{R}^{1+d}$, are transverse, where $\mathcal{F}$ denotes the space-time Fourier transform. Since the waves $e^{i t|\nabla|} f$ and $e^{i t|\nabla|} g$ propagate in the normal directions to these surfaces, the two waves can only interact strongly for short times. Thus we should expect the product $e^{i t|\nabla|} f e^{i t|\nabla|} g$ to decay faster than say $\left(e^{i t|\nabla|} f\right)^{2}$.

If we apply the above discussion to the bilinear estimate (1), since the normal direction to the cone is $\left(1,-\frac{\xi}{\mid \xi}\right)$, and the normal direction to the paraboloid is $(1,2 \xi)$, we should expect to improve on the range (2) obtained via the linear Strichartz estimates, by imposing a transversality condition of the form

$$
\left|\frac{\xi}{|\xi|}+2 \eta\right| \gtrsim 1
$$

for all $\xi \in \operatorname{supp} \widehat{f}$ and $\eta \in \widehat{g}$ (here $\widehat{f}$ denotes the spatial Fourier transform). Unfortunately, the simple transversality condition (4) does not suffice due to the lack of curvature of the cone along the surface of intersection

$$
\Sigma_{\text {wave }}(a, z)=\left\{(\tau, \xi) \in \operatorname{supp} \mathcal{F}\left[e^{i t|\nabla|} f\right] \mid(a, z)-(\tau, \xi) \in \operatorname{supp} \mathcal{F}\left[e^{i t \Delta} g\right]\right\}, \quad(a, z) \in \mathbb{R}^{1+d} .
$$

In fact it is well known that for certain surfaces, transversality alone is not sufficient to obtain the full bilinear range, see for instance [1] for the example of the hyperbolic paraboloid, and the related discussion in [3] [6]. However, imposing a stronger support condition gives the following.

Theorem 2 (Wave-Schrödinger bilinear restriction [6]). Let $d \geqslant 2,1 \leqslant q, r \leqslant 2$, and $\frac{2}{q}+\frac{d+1}{r}<d+1$. Let $\xi_{0}, \eta_{0} \in \mathbb{R}^{d}$ such that

$$
\left|\left(\frac{\xi_{0}}{\left|\xi_{0}\right|}+2 \eta_{0}\right) \cdot \frac{\xi_{0}}{\left|\xi_{0}\right|}\right| \gtrsim\left|\frac{\xi_{0}}{\left|\xi_{0}\right|}+2 \eta_{0}\right|
$$

and define $\lambda=\left|\eta_{0}\right|$, and $\alpha=\left|\frac{\xi_{0}}{\left|\xi_{0}\right|}+2 \eta_{0}\right|$. If

$$
\operatorname{supp} \widehat{f} \subset\left\{|\xi| \approx \lambda, \measuredangle\left(\xi, \xi_{0}\right) \ll \min \{1, \alpha\}\right\}, \quad \operatorname{supp} \widehat{g} \subset\left\{\left|\xi-\eta_{0}\right| \ll \alpha\right\}
$$

then we have

$$
\left\|e^{i t \Delta} f e^{i t|\nabla|} g\right\|_{L_{t}^{q} L_{x}^{r}\left(\mathbb{R}^{1+d}\right)} \lesssim(\min \{\alpha, \lambda, \alpha \lambda\})^{d+1-\frac{d+1}{r}-\frac{2}{q}} \alpha^{\frac{1}{r}-1} \lambda^{\frac{1}{q}-\frac{1}{2}}\|f\|_{L_{x}^{2}}\|g\|_{L_{x}^{2}} .
$$

Theorem 2 is a consequence of a bilinear restriction estimate for general phases obtained in [6]. The special case $q=r$ and $\alpha=\lambda=1$ could also be deduced from [3]. As the precise conditions in [6] are complicated, the derivation is slightly nontrival and we give the details below in Section 1 . The dependence on the parameters $\alpha$ and $\lambda$ is sharp, and this is particularly useful in applications to nonlinear PDE where $\alpha$ and $\lambda$ roughly correspond to a derivative loss/gain. Clearly, applying Sobolev embedding and interpolating with the trivial case $q=\infty, r=1$ can extend the range to $q, r \geqslant 2$ and $\frac{2}{q}+\frac{d+1}{r}<d+1$. However the dependence on $\alpha$ and $\lambda$ would no longer be sharp (i.e. losses may occur).

The condition (5) is necessary to obtain the full bilinear range $\frac{2}{q}+\frac{d+1}{r} \leqslant d+1$.

Theorem 3 (Transverse counter example). Suppose that the estimate (1) holds for all $f, g \in L^{2}\left(\mathbb{R}^{d}\right)$ with

$$
\operatorname{supp} \widehat{f} \subset\left\{\left|\xi-e_{1}\right| \ll 1\right\}, \quad \operatorname{supp} \widehat{g} \subset\left\{\left|2 \xi+e_{1}+e_{2}\right| \ll 1\right\} \text {. }
$$

Then

$$
\frac{2}{q}+\frac{d-1}{r}+\frac{1}{2 r} \leqslant d
$$

\footnotetext{
${ }^{2}$ Here $e_{j} \in \mathbb{R}^{d}, j=1, \ldots, n$ denote the standard basis vectors.
} 


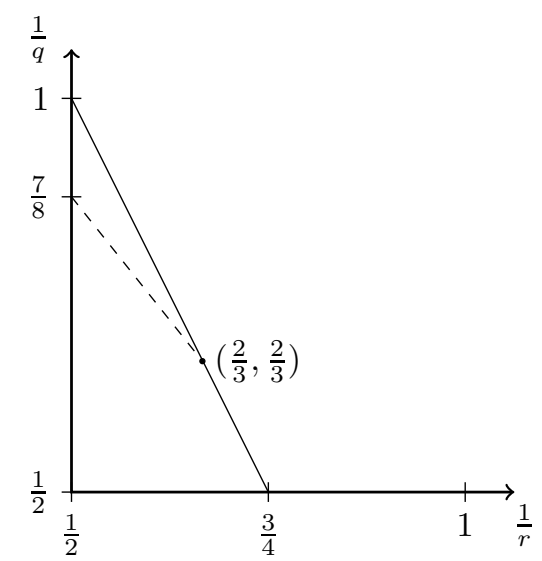

FiguRE 1 . The range of $1 \leqslant q, r \leqslant 2$ in $d=3$. The line corresponds to the sharp bilinear line $\frac{2}{q}+\frac{d+1}{r}=d+1$ given by Theorem 2 If (4) holds but (5) fails, then Theorem 3 states that the bilinear estimate (11) can only hold to the left of the dotted line.

Note that if we let $\xi_{0}=e_{1}$ and $\eta_{0}=-\frac{1}{2} e_{1}-\frac{1}{2} e_{2}$, then $\left|\frac{\xi_{0}}{\xi_{0} \mid}+2 \eta_{0}\right|=1$ but $\left(\frac{\xi_{0}}{\xi_{0} \mid}+2 \eta_{0}\right) \cdot \frac{\xi_{0}}{\left|\xi_{0}\right|}=0$. In other words the transversality condition (4) holds, but the stronger condition (5) fails. The range (6) is stronger than the bilinear range in Theorem 2 when $q$ is close to 1 and $d \leqslant 5$, see figure 1. If we drop the transversality condition completely, then a similar counter example can be used to prove the following.

Theorem 4 (Non-transverse counter example). Suppose that the estimate (1) holds for all $f, g \in L^{2}\left(\mathbb{R}^{d}\right)$ with $\operatorname{supp} \widehat{f}$, supp $\widehat{g} \subset\{|\xi| \approx 1\}$. Then

$$
\frac{2}{q}+\frac{d-1}{r} \leqslant d-\frac{1}{2}, \quad \frac{1}{q} \leqslant \frac{d+1}{4} .
$$

We give the proof of Theorem 3 and Theorem 4 in Section 2 below. In the positive direction, if (4) holds, but (5) fails, a naive adaption of the proof of Theorem 2 should give the region $\frac{2}{q}+\frac{d}{r}<d$. The loss of dimension corresponds to the lack of curvature in the radial direction (i.e. the cone only has $d-1$ directions of non-vanishing curvature). Note that there is a large gap between the potential range $\frac{2}{q}+\frac{d}{r}<d$ and the counter example given by Theorem 3. Similarly, even in the "linear" case when the transversality condition (4) is dropped, there is a gap between the counter example in Theorem 4 and the linear range given via Strichartz estimates (2). It is an interesting open question to determine the precise range of $(q, r)$ once the general condition (5) is dropped. In particular, it is not clear to the author what the optimal range for $(q, r)$ should be. Presumably the counter examples used in the proof of Theorem 3 and Theorem 4 can be improved.

In applications to nonlinear PDE, typically the homogeneous estimate in Theorem 2 is not sufficient, and it is more useful to have a version in suitable function spaces. One option is to work with $X^{s, b}$ type spaces. However, recently bilinear restriction estimates in the $U^{p}$ type spaces have proven useful, see for instance [7] and the discussion within. In the following we wish to give a general argument which can allow multilinear estimates for homogeneous solutions, to be upgraded to estimates in the adapted function spaces $U^{2}$. The underlying idea is straight forward. The first step is use the classical theorem of Marcinkiewicz-Zygmund that given a bound for a linear operator, a standard randomisation argument via Khintchine's inequality implies that a vector valued operator bound also holds. The second step is use the observation that a vector valued estimate immediately implies a $U^{2}$ bound, see for instance [6, Section 1.2] or [8, Remark 5.2]. As an example, we extend Theorem 2, and the multilinear restriction theorem 4 to $U^{2}$.

We start with the definition of $U^{2}$. A function $\phi \in L_{t}^{\infty} L_{x}^{2}$ is an atom if we can write $\phi(t)=\sum_{I} \mathbb{1}_{I}(t) g_{I}$, with the intervals $I \subset \mathbb{R}$ forming a partition of $\mathbb{R}$, and the $g_{I}: \mathbb{R}^{d} \rightarrow \mathbb{C}$ satisfying the bound

$$
\left(\sum_{I}\left\|g_{I}\right\|_{L_{x}^{2}}^{2}\right)^{\frac{1}{2}} \leqslant 1
$$


The atomic space $U^{2}$ is then defined as

$$
U^{2}=\left\{\sum_{j} c_{j} \phi_{j} \mid \phi_{j} \text { an atom and }\left(c_{j}\right) \in \ell^{1}\right\}
$$

with the induced norm

$$
\|u\|_{U^{2}}=\inf _{u=\sum_{j} c_{j} \phi_{j}} \sum_{j}\left|c_{j}\right|
$$

where the inf is over all representations of $u$ in terms of atoms. These spaces were introduced in unpublished work of Tataru, and studied in detail in [10, 9. To obtain the adapted function spaces $U_{|\nabla|}^{2}$ and $U_{\Delta}^{2}$ adapted to the wave and Schrödinger flows respectively, we define

$$
U_{|\nabla|}^{2}=\left\{u: \mathbb{R}^{1+d} \rightarrow \mathbb{C} \mid e^{-i t|\nabla|} u \in U^{2}\right\}, \quad U_{\Delta}^{2}=\left\{v: \mathbb{R}^{1+d} \rightarrow \mathbb{C} \mid e^{-i t \Delta} v \in U^{2}\right\} .
$$

Note that since $\mathbb{1}_{\mathbb{R}}(t) f \in U^{2}$, we clearly have $e^{i t|\nabla|} f \in U_{|\nabla|}^{2}$ and $e^{i t \Delta} f \in U_{\Delta}^{2}$. Thus the adapted function spaces contain all homogeneous solutions. Running the argument sketched above implies the following $U^{2}$ version of Theorem 2 ,

Theorem 5 (Wave-Schrödinger bilinear restriction in $U^{2}$ ). Let $d \geqslant 2,1 \leqslant q, r \leqslant 2$, and $\frac{2}{q}+\frac{d+1}{r}<d+1$. Let $\xi_{0}, \eta_{0} \in \mathbb{R}^{d}$ such that

$$
\left|\left(\frac{\xi_{0}}{\left|\xi_{0}\right|}+2 \eta_{0}\right) \cdot \frac{\xi_{0}}{\left|\xi_{0}\right|}\right| \gtrsim\left|\frac{\xi_{0}}{\left|\xi_{0}\right|}+2 \eta_{0}\right|
$$

and define $\lambda=\left|\eta_{0}\right|$, and $\alpha=\left|\frac{\xi_{0}}{\left|\xi_{0}\right|}+2 \eta_{0}\right|$. If

$$
\operatorname{supp} \widehat{u} \subset\left\{|\xi| \approx \lambda, \measuredangle\left(\xi, \xi_{0}\right) \ll \min \{1, \alpha\}\right\}, \quad \operatorname{supp} \widehat{v} \subset\left\{\left|\xi-\eta_{0}\right| \ll \alpha\right\}
$$

then we have

$$
\|u v\|_{L_{t}^{q} L_{x}^{r}\left(\mathbb{R}^{1+d}\right)} \lesssim(\min \{\alpha, \lambda, \alpha \lambda\})^{d+1-\frac{d+1}{r}-\frac{2}{q}} \alpha^{\frac{1}{r}-1} \lambda^{\frac{1}{q}-\frac{1}{2}}\|u\|_{U_{|\nabla|}^{2}}\|v\|_{U_{\Delta}^{2}} .
$$

Proof. Let $u=\sum_{I \in \mathcal{I}} e^{i t|\nabla|} f_{I}$ be a $U_{|\nabla|}^{2}$ atom, and let $v_{0}=e^{i t \Delta} g$ be a homogeneous solution to the Schrödinger equation. Assume that the support conditions (8) hold. Let $\left(\epsilon_{I}\right)_{I \in \mathcal{I}}$ be a family of independent, identically distributed random variables with $\epsilon_{I}=1$ with probability $\frac{1}{2}$, and $\epsilon_{I}=-1$ with probability $\frac{1}{2}$. The since the intervals $I$ are disjoint, we have via Khintchine's inequality

$$
|u| \leqslant\left(\sum_{I}\left|e^{i t|\nabla|} f_{I}\right|^{2}\right)^{\frac{1}{2}} \approx \mathbf{E}\left[\left|\sum_{I} \epsilon_{I} e^{i t|\nabla|} f_{I}\right|\right] .
$$

Therefore, since $q, r \geqslant 1$, applying Theorem 2 gives

$$
\begin{aligned}
\left\|u v_{0}\right\|_{L_{t}^{q} L_{x}^{r}} & \lesssim\left\|\mathbf{E}\left[\left|\sum_{I} \epsilon_{I} e^{i t|\nabla|} f_{I}\right|\right] v_{0}\right\|_{L_{t}^{q} L_{x}^{r}} \\
& \lesssim \mathbf{E}\left[\left\|e^{i t|\nabla|}\left(\sum_{I} \epsilon_{I} f_{I}\right) v_{0}\right\|_{L_{t}^{q} L_{x}^{r}}\right] \\
& \lesssim(\min \{\alpha, \lambda, \alpha \lambda\})^{d+1-\frac{d+1}{r}-\frac{2}{q}} \alpha^{\frac{1}{r}-1} \lambda^{\frac{1}{q}-\frac{1}{2}} \mathbf{E}\left[\left\|\sum_{I} \epsilon_{I} f_{I}\right\|_{L_{x}^{2}}\right]\|g\|_{L_{x}^{2}} .
\end{aligned}
$$

We now observe that Hölder's inequality, together with another application of Khintchine's inequality, implies that

$$
\mathbf{E}\left[\left\|\sum_{I} \epsilon_{I} f_{I}\right\|_{L_{x}^{2}}\right] \leqslant\left(\mathbf{E}\left[\left\|\sum_{I} \epsilon_{I} f_{I}\right\|_{L_{x}^{2}}^{2}\right]\right)^{\frac{1}{2}}=\left(\sum_{I}\left\|f_{I}\right\|_{L^{2}}^{2}\right)^{\frac{1}{2}}
$$

and consequently, applying the definition of the $U_{|\nabla|}^{2}$ norm, we obtain

$$
\left\|u v_{0}\right\|_{L_{t}^{q} L_{x}^{r}} \lesssim(\min \{\alpha, \lambda, \alpha \lambda\})^{d+1-\frac{d+1}{r}-\frac{2}{q}} \alpha^{\frac{1}{r}-1} \lambda^{\frac{1}{q}-\frac{1}{2}}\|u\|_{U_{|\nabla|}^{2} \mid}\|g\|_{L_{x}^{2}}
$$

To replace the homogeneous solution $v_{0}$ with a general $U_{\Delta}^{2}$ function follows by essentially repeating the above argument. In slightly more detail, suppose that $v=\sum_{J \in \mathcal{J}} e^{i t \Delta} g_{J}$ is a $U_{\Delta}^{2}$ atom, and let $\left(\epsilon_{J}\right)_{J \in \mathcal{J}}$ be a family 
of i.i.d. random variables with $\epsilon_{J}= \pm 1$ with equal probability. Then as above, but replacing Theorem 2 with (9), we see that

$$
\begin{aligned}
\|u v\|_{L_{t}^{q} L_{x}^{r}} & \lesssim\left\|u \mathbf{E}\left[\left|\sum_{J} \epsilon_{J} e^{i t \Delta} g_{J}\right|\right]\right\|_{L_{t}^{q} L_{x}^{r}} \\
& \lesssim \mathbf{E}\left[\left\|u \sum_{J} e^{i t \Delta} \epsilon_{J} g_{J}\right\| \|_{L_{t}^{q} L_{x}^{r}}\right] \\
& \lesssim(\min \{\alpha, \lambda, \alpha \lambda\})^{d+1-\frac{d+1}{r}-\frac{2}{q}} \alpha^{\frac{1}{r}-1} \lambda^{\frac{1}{q}-\frac{1}{2}}\|u\|_{U_{|\nabla|}^{2} \mid} \mathbf{E}\left[\left\|\sum_{J} \epsilon_{J} g_{J}\right\|_{L^{2}}\right] \\
& \lesssim(\min \{\alpha, \lambda, \alpha \lambda\})^{d+1-\frac{d+1}{r}-\frac{2}{q}} \alpha^{\frac{1}{r}-1} \lambda^{\frac{1}{q}-\frac{1}{2}}\|u\|_{U_{|\nabla|}^{2}}\left(\sum_{J}\left\|g_{J}\right\|_{L^{2}}^{2}\right)^{\frac{1}{2}} .
\end{aligned}
$$

Applying the definition the $U_{\Delta}^{2}$ norm, the required bound follows.

Strictly speaking the above theorem can also be obtain via the vector valued version of Theorem 2 from [6], see for instance [6, Section 1.2]. However the above alternative argument is more direct, and has the distinct advantage that it can be applied in more general situations. As an example, consider the following special case of the multilinear restriction theorem [4].

Theorem 6 (Multilinear restriction for Schrödinger [4]). Let $d \geqslant 2$ and $\epsilon>0$. Then for any $R \geqslant 1$ and any $f_{j} \in L^{2}\left(\mathbb{R}^{d}\right), j=1, \ldots, d$ with $\operatorname{supp} \widehat{f}_{j} \subset\left\{\left|\xi-e_{j}\right| \ll 1\right\}$ we have

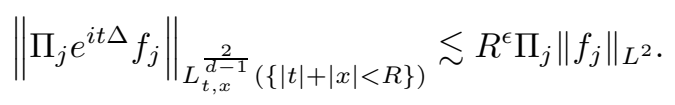

It is conjectured that the $R^{\epsilon}$ loss can be removed, but this is currently an open question (however see [1] and 2] for recent progress). The $U^{2}$ version of Theorem [6] is then the following.

Theorem 7 (Multilinear restriction for Schrödinger in $U^{2}$ ). Let $d \geqslant 2$ and $\epsilon>0$. Then for any $R \geqslant 1$ and any $u_{j} \in U_{\Delta}^{2}, j=1, \ldots, d$ with $\operatorname{supp} \widehat{u} \subset\left\{\left|\xi-e_{j}\right| \ll 1\right\}$ we have

$$
\left\|\Pi_{j} u_{j}\right\|_{L_{t, x}^{\frac{2}{d-1}(\{|t|+|x|<R\})}} \lesssim R^{\epsilon} \Pi_{j}\left\|u_{j}\right\|_{U_{\Delta}^{2}} .
$$

Proof. Let $B_{R}=\{|t|+|x|<R\}$. We proceed as in the proof of Theorem 5 . Thus suppose that $u_{1}=\sum_{I} e^{i t \Delta} f_{I}$ is $U_{\Delta}^{2}$ atom, and let $u_{j}^{0}=e^{i t \Delta} f_{j}$ for $j=2, \ldots, d$. Let $\epsilon_{I}$ be a family of i.i.d. random variables with $\epsilon_{I}= \pm 1$ with equal probability. An application of Khintchine's inequality implies that

$$
\left|u_{1}\right| \leqslant\left(\sum_{I}\left|e^{i t \Delta} f_{I}\right|^{2}\right)^{\frac{1}{2}} \approx\left(\mathbf{E}\left[\left|\sum_{I} \epsilon_{I} e^{i t|\nabla|} f_{I}\right|^{\frac{2}{d-1}}\right]\right)^{\frac{d-1}{2}}
$$

and hence Theorem 6 together with Hölder's inequality gives

$$
\begin{aligned}
& \left\|u_{1} \Pi_{j=2}^{d} u_{j}^{0}\right\|_{L_{t, x}^{\frac{d-1}{d-1}}\left(B_{R}\right)} \lesssim\left\|\left(\mathbf{E}\left[\left|\sum_{I} \epsilon_{I} e^{i t \Delta} f_{I}\right|^{\frac{2}{d-1}}\right]\right)^{\frac{d-1}{2}} \Pi_{j=2}^{d} u_{j}^{0}\right\|_{L_{t, x}^{\frac{d}{d-1}}\left(B_{R}\right)} \\
& \lesssim\left(\mathbf{E}\left[\left\|\sum_{I} \epsilon_{I} e^{i t \Delta} f_{I} \Pi_{j=2}^{d} u_{j}\right\|_{L_{t, x}\left(B_{R}\right)}^{\frac{2}{d-1}}\right]\right)^{\frac{2-1}{2}} \\
& \lesssim R^{\epsilon}\left(\mathbf{E}\left[\left\|\sum_{I} \epsilon_{I} f_{I}\right\|_{L^{2}}^{\frac{2}{d-1}}\right]\right)^{\frac{d-1}{2}} \Pi_{j=2}^{d}\left\|f_{j}\right\|_{L^{2}} \\
& \lesssim R^{\epsilon}\left(\mathbf{E}\left[\left\|\sum_{I} \epsilon_{I} f_{I}\right\|_{L^{2}}^{2}\right]\right)^{\frac{1}{2}} \Pi_{j=2}^{d}\left\|f_{j}\right\|_{L^{2}} \approx R^{\epsilon}\left(\sum_{I}\left\|f_{I}\right\|_{L^{2}}^{2}\right)^{\frac{1}{2}} \Pi_{j=2}^{d}\left\|f_{j}\right\|_{L^{2}}
\end{aligned}
$$

Applying the definition of the $U_{\Delta}^{2}$ norm, we conclude that

$$
\left\|u_{1} \Pi_{j=2}^{d} u_{j}^{0}\right\|_{L_{t, x}^{\frac{2}{d-1}\left(B_{R}\right)}} \lesssim R^{\epsilon}\left\|u_{1}\right\|_{U_{\Delta}^{2} \Pi_{j=2}^{d}\left\|f_{j}\right\|_{L^{2}} .}
$$


As in the proof of Theorem [5 repeating this argument with Theorem 6 replaced with (10) and $u_{1}$ replaced with $u_{2}$ gives

$$
\left\|u_{1} u_{2} \Pi_{j=3}^{d} u_{j}^{0}\right\|_{L_{t, x}^{d-1}\left(B_{R}\right)} \lesssim R^{\epsilon}\left\|u_{1}\right\|_{U_{\Delta}^{2}}\left\|u_{2}\right\|_{U_{\Delta}^{2}} \Pi_{j=3}^{d}\left\|f_{j}\right\|_{L^{2}} .
$$

The required bound follows by continuing in this manner.

We have not attempted to write down the most general transference type argument that can be deduced from the above arguments. However the underlying idea is simple; if a estimate holds for free solutions, then via randomisation it should hold in the vector valued case, and consequently it will also hold in $U^{2}$. Of course proving $U^{p}$ bounds, with $p \neq 2$ is substantially more challenging.

Acknowledgements. The author would like to thank Sebastian Herr and Kenji Nakanishi for a number of helpful discussions, as well as the University of Bielefeld and MATRIX for their kind hospitality while part of this work was conducted.

\section{Proof of Theorem 2}

It suffices to check the conditions in [6]. Suppose that $\xi_{0}, \eta_{0} \in \mathbb{R}^{d}$ such that (5) holds, and define $\lambda=\left|\eta_{0}\right|$, and $\alpha=\left|\frac{\xi_{0}}{\left|\xi_{0}\right|}+2 \eta_{0}\right|$. Let

$$
\Lambda_{1}=\left\{|\xi| \approx \lambda, \measuredangle\left(\xi, \xi_{0}\right) \ll \min \{1, \alpha\}\right\}, \quad \Lambda_{2}=\left\{\left|\xi-\eta_{0}\right| \ll \alpha\right\}
$$

and

$$
\Phi_{1}(\xi)=|\xi|, \quad \Phi_{2}(\xi)=-|\xi|^{2}, \quad \mathcal{H}_{1}=\lambda^{-1}, \quad \mathcal{H}_{1}=1 .
$$

In view of [6. Lemma 2.1 and Theorem 1.2], for $\{j, k\}=\{1,2\}$ and $\xi \in \Lambda_{j}, \eta \in \Lambda_{k}$, it suffices to check the following conditions:

(i) for all $v \in \mathbb{R}^{d}$ we have

$$
v \cdot\left(\nabla \Phi_{j}(\xi)-\nabla \Phi_{k}(\eta)\right)=0 \quad \Longrightarrow \quad\left|\nabla^{2} \Phi_{j}(\xi) v \wedge\left(\nabla \Phi_{j}(\xi)-\nabla \Phi_{k}(\eta)\right)\right| \gtrsim \mathcal{H}_{j} \alpha|v|,
$$

(ii) for $\xi^{\prime} \in \Lambda_{j}$ and $\eta^{\prime} \in \Lambda_{k}$ we have

$$
\left|\nabla \Phi_{j}(\xi)-\nabla \Phi_{j}\left(\xi^{\prime}\right)\right|+\left|\nabla \Phi_{k}(\eta)-\nabla \Phi_{k}\left(\eta^{\prime}\right)\right| \ll \alpha,
$$

(iii) the Hessian's satisfy

$$
\left|\nabla \Phi_{j}(\xi)-\nabla \Phi_{j}\left(\xi^{\prime}\right)-\nabla^{2} \Phi_{j}(\xi)\left(\xi-\xi^{\prime}\right)\right| \ll \mathcal{H}_{j}\left|\xi-\xi^{\prime}\right|,
$$

(iv) for $2<m \leqslant 5 d$ we have the derivative bounds

$$
\left\|\nabla^{m} \Phi_{j}\right\|_{L^{\infty}\left(\Lambda_{j}\right)}(\min \{\alpha, \lambda, \alpha \lambda\})^{m-2} \lesssim \mathcal{H}_{j}, \quad \mathcal{H}_{j} \min \{\alpha, \lambda, \alpha \lambda\} \lesssim \alpha .
$$

(v) we have the surface measure condition

$$
\sup _{(a, h) \in \mathbb{R}^{1+d}} \sigma_{d-1}\left(\left\{\xi \in \Lambda_{2} \cap\left(h-\Lambda_{1}\right) \mid \Phi_{2}(\xi)+\Phi_{1}(h-\xi)=a\right\}\right) \lesssim(\min \{\alpha, \lambda, \alpha \lambda\})^{d-1}
$$

where $\sigma_{d-1}$ is the induced Lebesgue surface measure.

To check the first property (i), by unpacking the definition, our goal is to show that for any $\xi \in \Lambda_{1}$ and $\eta \in \Lambda_{2}$ we have

$$
z \cdot(\omega+2 \eta)=0 \quad \Longrightarrow \quad|(z-(\omega \cdot z) \omega) \wedge(\omega+2 \eta)| \gtrsim|z||\omega+2 \eta|,
$$

where $\omega=\frac{\xi}{|\xi|}$. In view of the definition of the sets $\Lambda_{j}$ we have

$$
|(\omega+2 \eta) \cdot \omega| \gtrsim|\omega+2 \eta|
$$

and hence as $(z \cdot \omega)(\omega+2 \eta) \cdot \omega=2 z \cdot(\eta-(\eta \cdot \omega) \omega)$ we get

Therefore

$$
|z \cdot \omega| \leqslant \frac{2|z \cdot(\eta-(\eta \cdot \omega) \omega)|}{|(\omega+2 \eta) \cdot \omega|} \lesssim|z-(\omega \cdot z) \omega|
$$

$$
|(z-(\omega \cdot z) \omega) \wedge(\omega+2 \eta)| \geqslant|z-(\omega \cdot z) \omega||(\omega+2 \eta) \cdot \omega| \gtrsim|z||\omega+2 \omega|
$$

as required. 
The properties (ii), ..., (iv) follow by direct computation. Finally, to check the surface measure condition (v), we note that the vector $N=\frac{\xi_{0}}{\left|\xi_{0}\right|}+2 \eta_{0}$ is essentially normal to the surface. On the other hand, from (5),$N$ is roughly pointing in the direction $\frac{\eta_{0}}{\left|\eta_{0}\right|}$. Hence the surface measure can be bounded by projecting onto the plane orthogonal to $\frac{\eta_{0}}{\mid \eta_{0}}$. Since this projection is contained in a ball of radius $\lesssim \min \{\alpha, \lambda, \alpha \lambda\}$, the bound follows.

\section{Counter Examples}

We first observe that by a randomisation argument, if the estimate (1) holds for all $f, g \in L^{2}$ with $\operatorname{supp} \widehat{f} \subset \Lambda_{1} \subset \mathbb{R}^{n}$ and $\operatorname{supp} \widehat{g} \subset \Lambda_{2}$, then in fact we also have the vector valued version

$$
\left\|\left(\sum_{j}\left|e^{i t|\nabla|} f_{j}\right|^{2}\right)^{\frac{1}{2}}\left(\sum_{k}\left|e^{i t \Delta} g_{k}\right|^{2}\right)^{\frac{1}{2}}\right\|_{L_{t}^{q} L_{x}^{r}} \lesssim\left(\sum_{j}\left\|f_{j}\right\|_{L^{2}}^{2}\right)^{\frac{1}{2}}\left(\sum_{k}\left\|g_{k}\right\|_{L_{x}^{2}}^{2}\right)^{\frac{1}{2}}
$$

for all supp $\widehat{f}_{j} \subset \Lambda_{1}$, supp $\widehat{g}_{k} \subset \Lambda_{2}$. This follows by noting that if $\epsilon_{j}$ is an i.i.d. family of random variables with $\epsilon_{j}= \pm$ with equal probability, then as in the proof of Theorem 5 , we have via Khintchine's inequality and (1)

$$
\begin{aligned}
\left\|\left(\sum_{j}\left|e^{i t|\nabla|} f_{j}\right|^{2}\right)^{\frac{1}{2}} e^{i t \Delta} g\right\|_{L_{t}^{q} L_{x}^{r}} & \approx\left\|\mathbf{E}\left[\left|\sum_{j} \epsilon_{j} e^{i t|\nabla|} f_{j}\right|\right] e^{i t \Delta} g\right\|_{L_{t}^{q} L_{x}^{r}} \\
& \lesssim \mathbf{E}\left[\left\|\sum_{j} \epsilon_{j} e^{i t|\nabla|} f_{j} e^{i t \Delta} g\right\|_{L_{t}^{q} L_{x}^{r}}\right] \\
& \lesssim \mathbf{E}\left[\left\|\sum_{j} \epsilon_{j} e^{i t|\nabla|} f_{j}\right\|_{L_{x}^{2}}\right]\|g\|_{L_{x}^{2}} \\
& \lesssim \mathbf{E}\left[\left\|\sum_{j} \epsilon_{j} e^{i t|\nabla|} f_{j}\right\|_{L_{x}^{2}}^{2}\right]^{\frac{1}{2}}\|g\|_{L_{x}^{2}} \approx\left(\sum_{j}\left\|f_{j}\right\|_{L^{2}}^{2}\right)^{\frac{1}{2}}\|g\|_{L^{2}} .
\end{aligned}
$$

Repeating this argument for the Schrödinger component then gives (11). Consequently, we see that the scalar version (1) holds, if and only if the vector valued version (11) holds. Thus to prove Theorem 3 and Theorem 4, it suffices to obtain vector valued counter examples.

2.1. Proof of Theorem [3. Let $N \geqslant 1$ and $\widehat{f}, \widehat{g} \in C_{0}^{\infty}$ with

$$
\operatorname{supp} \widehat{f} \subset\left\{\left|\xi_{1}-1\right| \ll 1,\left|\xi^{\prime}\right| \ll N^{-1}\right\}, \quad \operatorname{supp} \widehat{g} \subset\left\{\left|2 \xi-e_{1}-e_{2}\right| \ll N^{-\frac{1}{2}}\right\}
$$

and $\|f\|_{L^{2}} \approx N^{\frac{d-1}{2}},\|g\|_{L^{2}} \approx N^{\frac{d}{4}}$. A short computation using integration by parts shows that we can choose $f, g$ such that

$$
|u(t, x)|=\left|e^{i t|\nabla|} f(x)\right| \geqslant 1 \quad \text { for all } \quad|t| \leqslant N^{2},\left|x_{1}+t\right| \leqslant 1,\left|x^{\prime}\right| \leqslant N
$$

and

$$
|v(t, x)|=\left|e^{i t \Delta} g(x)\right| \geqslant 1 \quad \text { for all } \quad|t| \leqslant N^{1},\left|x_{1}+t\right| \leqslant N^{\frac{1}{2}},\left|x_{2}+t\right| \leqslant N^{\frac{1}{2}},\left|x^{\prime \prime}\right| \leqslant N^{\frac{1}{2}}
$$

where we write $x=\left(x_{1}, x^{\prime}\right)=\left(x_{1}, x_{2}, x^{\prime \prime}\right)$. In other words the free wave $u$ is $\geqslant 1$ on a plate of dimension $N^{2} \times 1 \times N^{d-1}$ oriented in the $\left(1,-e_{1}\right)$ direction, with short direction $e_{1}$, while the free Schrödinger wave $v$ is $\geqslant 1$ on a tube of dimensions $N \times N^{\frac{d}{2}}$ oriented in the $\left(1,-e_{1}-e_{2}\right)$ direction. Define the set

$$
\Omega=\left\{|t| \leqslant N^{-2},\left|x_{1}+t\right| \leqslant N^{\frac{1}{2}},\left|x^{\prime}\right| \leqslant N\right\} .
$$

The support properties of $u$, implies that for any $(t, x) \in \Omega$ we have

$$
U(t, x)=\left(\sum_{\substack{j \in \mathbb{Z} \\|j| \leqslant N^{\frac{1}{2}}}}\left|u\left(t, x+j e_{1}\right)\right|^{2}\right)^{\frac{1}{2}} \gtrsim 1 .
$$


Similarly, translating the free Schrödinger wave in both space and time gives for any $(t, x) \in \Omega$

$$
V(t, x)=\left(\sum_{\substack{\left(j_{2}, \ldots, j_{d}\right) \in \mathbb{Z}^{d-1} \\\left|j_{1}\right|, \ldots,\left|j_{d}\right| \lesssim N^{\frac{1}{2}}}} \sum_{\substack{k \in \mathbb{Z} \\|k| \leqslant N}}\left|v\left(t+N k, x+N^{\frac{1}{2}}\left(j_{2} e_{2}+\cdots+j_{d} e_{d}\right)\right)\right|^{2}\right)^{\frac{1}{2}} \gtrsim 1 .
$$

Since the wave and Schrödinger equations are translation invariant, the bound (11) implies that

$$
\begin{aligned}
N^{\frac{2}{q}} N^{\frac{d-1}{r}+\frac{1}{2 r}} \lesssim\left\|\mathbb{1}_{\Omega}\right\|_{L_{t}^{q} L_{x}^{r}} & \lesssim\|U V\|_{L_{t}^{q} L_{x}^{r}} \\
& \lesssim\left(\sum_{|j| \leqslant N^{\frac{1}{2}}}\|f\|_{L^{2}}\right)^{\frac{1}{2}}\left(\sum_{\left|j_{1}\right|, \ldots,\left|j_{d}\right| \lesssim N^{\frac{1}{2}}} \sum_{|k| \leqslant N}\|g\|_{L^{2}}^{2}\right)^{\frac{1}{2}} \lesssim N^{\frac{d-1}{2}+\frac{1}{4}} \times N^{\frac{d}{2}+\frac{1}{4}} .
\end{aligned}
$$

Letting $N \rightarrow \infty$, we see that this is only possible if

$$
\frac{2}{q}+\frac{d-1}{r}+\frac{1}{2 r} \leqslant d
$$

2.2. Proof of Theorem 4, Let $1 \leqslant M \leqslant N$ and $\widehat{f}, \widehat{g} \in C_{0}^{\infty}$ with

$$
\operatorname{supp} \widehat{f} \subset\left\{\left|\xi_{1}-1\right| \ll 1,\left|\xi^{\prime}\right| \ll N^{-1}\right\}, \quad \operatorname{supp} \widehat{g} \subset\left\{\left|2 \xi-e_{1}\right| \ll M^{-1}\right\}
$$

and $\|f\|_{L^{2}} \approx N^{\frac{d-1}{2}},\|g\|_{L^{2}} \approx M^{\frac{d}{2}}$. A short computation using integration by parts shows that we can choose $f, g$ such that

$$
|u(t, x)|=\left|e^{i t|\nabla|} f(x)\right| \geqslant 1 \quad \text { for all } \quad|t| \leqslant N^{2},\left|x_{1}+t\right| \leqslant 1,\left|x^{\prime}\right| \leqslant N
$$

and

$$
|v(t, x)|=\left|e^{i t \Delta} g(x)\right| \geqslant 1 \quad \text { for all } \quad|t| \leqslant M^{1},\left|x_{1}+t\right| \leqslant M,\left|x^{\prime}\right| \leqslant M .
$$

In other words the free wave $u$ is $\geqslant 1$ on a plate of dimension $N^{2} \times 1 \times N^{d-1}$ oriented in the $\left(1,-e_{1}\right)$ direction, with short direction $e_{1}$, while the free Schrödinger wave $v$ is $\geqslant 1$ on a tube of dimensions $M^{2} \times M^{d}$ oriented in the $\left(1,-e_{1}\right)$ direction. Similar to the proof of Theorem (3), we consider a number of temporal translated Schrödinger waves covering the set

$$
\Omega=\left\{|t| \leqslant N^{-2},\left|x_{1}+t\right| \leqslant 1,\left|x^{\prime}\right| \leqslant M\right\} .
$$

More precisely, we have for all $(t, x) \in \Omega$

$$
V(t, x)=\left(\sum_{\substack{j \in \mathbb{Z} \\|j| \leqslant \frac{N^{2}}{M^{2}}}}\left|v\left(t+j M^{2}, x\right)\right|^{2}\right)^{\frac{1}{2}} \gtrsim 1
$$

Since we clearly have $|u| \geqslant 1$ on $\Omega$ by construction, we see that if (1) holds, then the vector valued version (11) holds, and hence

$$
N^{\frac{2}{q}} M^{\frac{d-1}{r}} \lesssim\left\|\mathbb{1}_{\Omega}\right\|_{L_{t}^{q} L_{x}^{r}} \lesssim\|u V\|_{L_{t}^{q} L_{x}^{r}} \lesssim\|f\|_{L^{2}}\left(\sum_{|j| \leqslant \frac{N^{2}}{M^{2}}}\|g\|_{L^{2}}^{2}\right)^{\frac{1}{2}} \lesssim N^{\frac{d-1}{2}} \times N M^{\frac{d}{2}-1}
$$

Rearranging, we see that we must have

$$
N^{\frac{2}{q}-\frac{d+1}{2}} M^{\frac{d-1}{r}-\frac{d-2}{2}} \lesssim 1
$$

Letting $M=1$ and $N \rightarrow \infty$ gives the restriction

$$
\frac{1}{q} \leqslant \frac{d+1}{4}
$$

On the other hand, letting $M=N \rightarrow \infty$, gives the condition

$$
\frac{2}{q}+\frac{d-1}{r} \leqslant d-\frac{1}{2}
$$




\section{REFERENCES}

1. Ioan Bejenaru, The almost optimal multilinear restriction estimate for hypersurfaces with curvature: the case of $n-1$ hypersurfaces in $\mathbb{R}^{n}$, arXiv:2002.12488 [math.CA] .

2. - The multilinear restriction estimate: almost optimality and localization, arXiv:1912.06664 [math.CA].

3. Ioan Bejenaru, Optimal bilinear restriction estimates for general hypersurfaces and the role of the shape operator, International Mathematics Research Notices 2017 (2017), no. 23, 7109-7147.

4. Jonathan Bennett, Anthony Carbery, and Terence Tao, On the multilinear restriction and Kakeya conjectures, Acta Math. 196 (2006), no. 2, 261-302. MR 2275834

5. J. Bourgain, Estimates for cone multipliers, Geometric aspects of functional analysis (Israel, 1992-1994), Oper. Theory Adv. Appl., vol. 77, Birkhäuser, Basel, 1995, pp. 41-60. MR 1353448

6. Timothy Candy, Multi-scale bilinear restriction estimates for general phases, Mathematische Annalen 375 (2019), no. 1-2, $777-843$.

7. Timothy Candy and Sebastian Herr, On the division problem for the wave maps equation, Annals of PDE 4 (2018), no. 2, 17.

8. _ Transference of bilinear restriction estimates to quadratic variation norms and the Dirac-Klein-Gordon system, Anal. PDE 11 (2018), no. 5, 1171-1240.

9. Martin Hadac, Sebastian Herr, and Herbert Koch, Well-posedness and scattering for the KP-II equation in a critical space, Ann. Inst. H. Poincaré Anal. Non Linéaire 26 (2009), no. 3, 917-941. MR 2526409 (2010d:35301)

10. Herbert Koch and Daniel Tataru, Dispersive estimates for principally normal pseudodifferential operators, Comm. Pure Appl. Math. 58 (2005), no. 2, 217-284. MR 2094851 (2005m:35323)

11. Sanghyuk Lee, Bilinear restriction estimates for surfaces with curvatures of different signs, Trans. Amer. Math. Soc. $\mathbf{3 5 8}$ (2006), no. 8, 3511-3533 (electronic). MR 2218987 (2007a:42023)

12. T. Tao and A. Vargas, A bilinear approach to cone multipliers. I. Restriction estimates, Geom. Funct. Anal. 10 (2000), no. 1, 185-215. MR 1748920 (2002e:42012)

13. Terence Tao, Endpoint bilinear restriction theorems for the cone, and some sharp null form estimates, Math. Z. 238 (2001), no. 2, 215-268. MR 1865417 (2003a:42010)

14. Daniel Tataru, Null form estimates for second order hyperbolic operators with rough coefficients, Harmonic analysis at Mount Holyoke (South Hadley, MA, 2001), Contemp. Math., vol. 320, Amer. Math. Soc., Providence, RI, 2003, pp. 383409. MR 1979953

15. Faruk Temur, An endline bilinear cone restriction estimate for mixed norms, Math. Z. 273 (2013), no. 3-4, 1197-1214. MR 3030696

16. Thomas Wolff, A sharp bilinear cone restriction estimate, Ann. of Math. (2) 153 (2001), no. 3, 661-698. MR 1836285 (2002j:42019)

17. Vladimir E. Zakharov, Collapse of Langmuir waves, Sov. Phys. JETP 35 (1972), no. 5, 908-914.

(T. Candy) Department of Mathematics and Statistics, University of Otago, Po Box 56, Dunedin 9054, New ZEALAND

E-mail address: tcandy@maths.otago.ac.nz 